\title{
Diferencias individuales en razonamiento deductivo: una revisión narrativa
}

\author{
María del Carmen Crivello*, Guillermo Macbeth**, Mauro Fioramonti***, \\ Eugenia Razumiejczyk ${ }^{* * * *}$
}

* Becaria doctoral del Consejo Nacional de Investigaciones Científicas y Técnicas de Argentina, Facultad de Ciencias de la Educación, Universidad Nacional de Entre Ríos, Provincia de Entre Ríos, Argentina Correo electrónico: mcrivello@hotmail.com

** Doctor en Psicología. Investigador adjunto del Consejo Nacional de Investigaciones Científicas y Técnicas de Argentina, Facultad de Ciencias de la Educación, Universidad Nacional de Entre Ríos, Provincia de Entre Ríos, Argentina Correo electrónico: g.macbeth@conicet.gov.ar

*** Becario doctoral del Consejo Nacional de Investigaciones Científicas y Técnicas de Argentina, Facultad de Ciencias de la Educación, Universidad Nacional de Entre Ríos, Provincia de Entre Ríos, Argentina. Correo electrónico: mauro.fdd@gmail.com

****Doctora en Psicología. Investigadora asistente del Consejo Nacional de Investigaciones Científicas y Técnicas de Argentina, Facultad de Ciencias de la Educación, Universidad Nacional de Entre Ríos, Provincia de Entre Ríos, Argentina Correo electrónico: eugeniaraz@hotmail.com

Recibido: 9 de noviembre del 2015 Aprobado: 8 de marzo del 2016

Cómo citar este artículo: Crivello, M., Macbeth, G., Fioramonti, M. y Razumiejczyk, E. (2016). Diferencias individuales en razonamiento deductivo: una revisión narrativa. Pensando Psicología, 12(19), 23-38. doi: http://dx.doi.org/10.16925/ pe.v12i19.1326

\section{Resumen}

Introducción: el presente estudio aborda la cuestión de diferencias individuales en razonamiento deductivo. En él se tratan algunas de las variables relacionadas con el campo: las habilidades cognitivas, el desarrollo, el género y la cultura de las personas. Objetivo: se pretende ofrecer una revisión narrativa de las principales propuestas dentro del área, con especial atención a los factores sociales. Metodología: se realiza un análisis cualitativo de la bibliografía existente, relevando aportes principalmente clásicos, y algunos recientes. Resultados: han sido detectadas variables de diferencias individuales que pueden contribuir a una mejor comprensión del fenómeno bajo estudio. Conclusión: se presentan de manera sintética los principales hallazgos y limitaciones del trabajo, relacionadas estas con la combinación de evidencia en futuros estudios metaanalíticos.

Palabras clave: desarrollo, diferencias individuales, género y cultura, inteligencia, razonamiento. 


\title{
Individual Differences in Deductive Reasoning: A Narrative Review
}

\begin{abstract}
Introduction: This study addresses the issue of individual differences in deductive reasoning. It covers some of the variables related to the field: cognitive skills, development, gender and culture of people. Objective: To provide a narrative review of the main proposals in the area, emphasizing social factors. Methodology: A qualitative analysis of existing literature is performed, assessing mainly classic and some recent contributions. Results: Variables of individual differences that can contribute to a better understanding of the phenomenon under study have been detected. Conclusion: The main findings and limitations of the work, related to the combination of evidence in future meta-analytic studies, were summarized.
\end{abstract}

Keywords: development, individual differences, gender and culture, intelligence, reasoning.

\section{Diferenças individuais em raciocínio dedutivo: uma revisão narrativa}

\section{Resumo}

Introdução: este estudo aborda a questão de diferenças individuais em raciocínio dedutivo. No texto, tratam-se de algumas variáveis relacionadas com o campo: as habilidades cognitivas, o desenvolvimento, o gênero e a cultura das pessoas. Objetivo: pretende-se oferecer uma revisão narrativa das principais propostas dentro da área, com especial atenção aos fatores sociais. Metodologia: realiza-se uma análise qualitativa da bibliografia existente, relevando contribuições principalmente clássicas e algumas recentes. Resultados: detectaram-se variáveis de diferenças individuais que podem contribuir para uma melhor compreensão do fenômeno em estudo. Conclusão: apresentam-se de maneira sintética os principais achados e limitações do trabalho, relacionados com a combinação de evidência em futuros estudos meta-analíticos.

Palavras-chave: desenvolvimento, diferenças individuais, gênero e cultura, inteligência, raciocínio. 


\section{Introducción}

En psicología del razonamiento, un punto de partida común suele ser el reconocimiento del hombre como un ser racional, afirmación con frecuencia atribuida a Aristóteles (Baron, 2008), y la adjudicación consecuente al ser humano de una capacidad única para razonar. Según Johnson-Laird (1983), dicha facultad se encuentra en la esencia misma de la mentalidad humana, encontrándose en el desarrollo de la ciencia, la cultura y la tecnología, el máximo exponente de ello.

Dada la preponderancia que el pensar tiene sobre la vida del hombre, no sorprende observar el creciente interés en el estudio del razonamiento humano. Sin embargo, ¿a qué se hace referencia cuando se habla de razonamiento deductivo? Según Monserrat y Valiña (2002), razonamiento deductivo es el proceso por el cual se parte de varias premisas para llegar a una única conclusión, aunque en ocasiones sea posible el paso inmediato de una única premisa a una única conclusión. Asimismo, si bien las proposiciones suelen ser expresadas verbalmente, en el caso de algunas inferencias prácticas, las premisas pueden consistir en un estado de cosas percibido o imaginado, y la conclusión en un curso de acción. Finalmente, cuando se razona, el objetivo es llegar a conclusiones que sean verdaderas, o al menos probables - dadas las premisas-, pero sobre todo, a conclusiones que sean novedosas y mantengan coherencia (JohnsonLaird y Byrne, 1991).

Una de las tareas de los científicos cognitivos es, en consecuencia, analizar qué inferencias son racionales, cuáles son los procesos mentales que realizan estas inferencias y cómo se implementan estos procesos en el cerebro (Johnson-Laird, 1995). En este contexto, una de las teorías que ha logrado explicar con éxito una amplia gama de fenómenos del razonamiento es la Teoría de Modelos Mentales o ммт (Macbeth et al., 2014). Dicha teoría, planteada por JohnsonLaird (1983) y reformulada por Johnson-Laird y Byrne (1991), surge en oposición a las teorías tradicionales de la forma lógica (Macbeth, Razumiejczyk, y Crivello, 2014), cuyo principal postulado plantea que la razón humana depende de reglas formales de inferencia similares a las de un cálculo lógico (JohnsonLaird, 2010). La ммт por su parte, sostiene que los fenómenos de razonamiento son semánticamente impulsados, en lugar de estar basados en reglas lógicas (Johnson-Laird, 1983). Para esta, los sujetos razonan manipulando modelos mentales. Estos harían referencia a representaciones semánticas y relaciones sintácticas de objetos y situaciones del mundo real, elaboradas por los sujetos a partir de la información descrita en las premisas, así como también de su propio conocimiento del mundo (Monserrat y Valiña, 2002).

Según este modelo, cuando los seres humanos perciben el mundo, la visión obtiene un modelo mental respecto de cómo son las cosas que aparecen en escena. De igual modo, cuando los sujetos comprenden el mundo, construyen una representación similar aunque menos rica- de este, un modelo mental basado en la percepción del mundo y sus conocimientos (Johnson-Laird, 1983). Paralelamente, la teoría plantea tres supuestos principales en relación con este proceso. En primer lugar, postula que cada modelo mental representa lo que es común a un conjunto distinto de posibilidades. En segundo lugar, dice que los modelos mentales son tan icónicos como pueden serlo. En tercer lugar, plantea que estos representan lo que es verdadero y omiten lo que es falso. Finalmente, para la ммт, la dificultad en un razonamiento estaría modulada por la cantidad de modelos mentales que el sujeto necesite para generar una conclusión (Johnson-Laird, 2010).

Otra teoría con amplio desarrollo dentro del campo de la psicología del razonamiento es la teoría de doble procesamiento (Stanovich y West, 2000; Evans, 2003; 2008; Evans y Stanovich, 2013, entre otros). En general, las teorías ubicadas en este marco coinciden en plantear dos sistemas diferentes para explicar el razonamiento humano, en lugar de un único mecanismo universal de inferencia. Estos sistemas presentarían diferencias en aspectos funcionales, velocidad de procesamiento, acceso a la conciencia y capacidad computacional demandada (Seoane, Valiña, Rodríguez, Montserrat y Ferraces, 2007). Tal como afirma De Neys (2006), las teorías de doble procesamiento vienen de muchos colores, sin embargo, se asume cierto consenso a la hora de señalar la existencia de un sistema 1 , o heurístico, caracterizado como intuitivo, rápido y de bajo consumo atencional; en co-existencia con un sistema 2, o analítico, descripto como reflexivo, lento y de alto consumo atencional (Schroyens, Schaeken, Fias, y d'Ydewalle, 2000). Así, las respuestas ofrecidas por el sistema 1 se distinguirían por ser más intuitivas y con tendencia a basarse en la experiencia personal, las asociaciones y creencias previas, al tiempo que el sistema 2 sería más deliberativo, formal y con tendencia a razonar de acuerdo con formas lógicas (Stanovich y West, 2000; 
Newstead, Handley, Harley, Wright y Farrelly, 2004). No obstante, resulta oportuno aclarar que la distinción entre estos dos sistemas rige para un conjunto de teorías de doble procesamiento, de las cuales no todas son convergentes (Newstead, 2000; Evans y Stanovich, 2013).

Por otro lado, dicha teoría asume que con frecuencia ambos sistemas tenderían a trabajar en concierto. En tales circunstancias, el sistema heurístico actuaría por defecto, proporcionando conclusiones rápidas, frugales y correctas. Pese a lo anterior, también puede ocurrir que el sistema heurístico actúe de modo preponderante, ofreciendo respuestas sesgadas a situaciones que requerirían un razonamiento más detenido. En tales oportunidades, ambos sistemas entrarían en conflicto. Dado el mencionado caso, el sistema analítico necesitará actuar anulando la respuesta del sistema heurístico, ofreciendo a cambio una respuesta más adecuada. Así, el sistema heurístico supondría operar con rapidez y de forma automática, al tiempo que las operaciones del sistema analítico serían más lentas y exigentes de los recursos computacionales de la persona (De Neys, 2006).

De la mano de lo anterior, desarrollos científicos recientes (Stanovich y West, 2000; Evans, 2003; Newstead et al., 2004; Seoane et al., 2007; Avery, Smillie, y de Fockert, 2013) plantean una correlación directa entre la capacidad de la memoria operativa y la eficacia del procesamiento analítico, y medidas de inteligencia general. Ligado a este punto, se encuentra el campo de investigación que relaciona las diferencias individuales y el razonamiento, a partir de los influyentes aportes de Stanovich y West (2000).

La afirmación según la cual las personas aprenden y piensan de modos diferentes parece un asunto bastante evidente (Sternberg, Grigorenko y Zhang, 2008). Sin embargo, el estudio de las diferencias individuales en el ámbito de la psicología del razonamiento ha pasado de ser un aspecto prácticamente obviado, a ser uno de los temas que mayor investigación ha generado en las últimas décadas (Galián, Carranza, Escudero, Ato y Ato, 2006). Por diferencias individuales, se entiende aquellas variables atribuibles a las características propias del sujeto, más allá de las relacionadas con el contexto y contenido de la tarea. En este marco, el propósito de la presente contribución es realizar una revisión de aquellas diferencias identificadas como mayormente relevantes. En primer lugar, se analizan dos de las variables más antiguas dentro del campo: aquellas relacionadas con las habilidades cognitivas y la disposición al conocimiento. Posteriormente, se abordarán otras variables de menor desarrollo pero igual relevancia en el actual debate: aquellas relacionadas con el desarrollo, el género y la cultura de las personas.

\section{Diferencias individuales en razonamiento deductivo}

Una cantidad sustancial de literatura científica ha establecido que, con frecuencia, las respuestas de las personas en tareas de razonamiento serían propensas a desviarse de la actuación considerada normativa (Stanovich y West 2000; Stanovich, 2012). El hecho de que las personas evalúen probabilidades de modo incorrecto, no calibren correctamente sus grados de creencia, dejen afectar sus decisiones por un contexto irrelevante y muestren muchos otros sesgos en el procesamiento de la información, sería un claro ejemplo de ello (Gilovich, Griffin y Kahneman, 2002; Baron, 2008; Stanovich, 2012). En este sentido, observar cómo responden las personas ante determinadas tareas controladas conforma lo que comúnmente se conoce como "modelos descriptivos". Dichos modelos son el objetivo de la mayor parte de la psicología empírica. En contraste con los modelos descriptivos - los cuales se ocupan de la conducta observada-, los "modelos normativos" expresan estándares de rendimiento cognitivo. Se argumenta que cumplir estos estándares sirve para optimizar la precisión de las creencias y eficacia en las diferentes tareas estudiadas (Stanovich y West, 1998).

Durante muchos años, el eje central en el campo de la heurística y los sesgos en el razonamiento humano giró en torno a demostrar cómo los modelos descriptivos de la conducta humana a menudo diferían de los modelos normativos. En este contexto, numerosos estudios han detectado la existencia de ciertas tareas de razonamiento en las que todas las personas, sin una formación o instrucción especial, ofrecían casi invariablemente - igual respuesta (Stanovich, 2012). Pese a ello, lo que ha sido ignorado dentro del campo es que, si bien con frecuencia la persona promedio tiende a demostrar un exceso de confianza al elegir las opciones inadecuadas en las tareas de selección, al cometer la falacia de la conjunción, y así sucesivamente en cada uno de los experimentos considerados clásicos dentro de la heurística, algunas otras personas, sin embargo, son capaces de ofrecer la respuesta normativa estándar, es decir, la respuesta esperada (Stanovich y West, 2000). En otras palabras, de lo que se ha hecho caso omiso en este gran 
debate de la racionalidad, es de la existencia de diferencias individuales entre personas (Stanovich, 2012). Se ha privilegiado lo nomotético sobre lo idiográfico (Barlow y Nock, 2009).

Tal como fue señalado, el estudio de las diferencias individuales en el ámbito de la psicología del razonamiento se ha convertido en un tema de creciente interés en la actualidad. Pese a la vigencia que el debate todavía conserva, numerosos avances han sido posibles. A continuación, se ofrece un breve recorrido por estos.

\section{Dos niveles de análisis en diferencias individuales: nivel algorítmico y nivel intencional}

Desde sus inicios, el estudio de diferencias individuales en razonamiento deductivo estuvo fundamentalmente ligado a procesos cognitivos, estudiados por investigadores del procesamiento de la información interesados en las bases cognitivas de la ejecución de tests (Stanovich y West, 1997). Dichos teóricos consideraban que estas bases subyacían a la denominada inteligencia psicométrica general. En la actualidad, esta postura tiene aún numerosos adherentes. Desde esta perspectiva, la variación en la habilidad cognitiva supone diferencias individuales en la eficacia de procesamiento, pero diferencias a un nivel que los teóricos de la ciencia cognitiva denominan algorítmico (Seoane et al., 2007). Siguiendo esta línea, la magnitud de la correlación entre el desempeño en una tarea de razonamiento y la capacidad cognitiva, proporcionaría una pista empírica sobre la importancia de las limitaciones algorítmicas al momento de explicar las discrepancias entre los modelos descriptivos y normativos (Stanovich y West, 1998).

La capacidad cognitiva ha sido uno de los constructos más estudiados dentro de la psicología. Décadas de investigaciones empíricas han mostrado correlaciones directas entre esta y otras medidas de rendimiento psicológico (Macpherson y Stanovich, 2007). Así, pareciera que - al menos en grado moderado- las discrepancias entre el rendimiento real y los modelos normativos serían explicadas por la variación en las limitaciones computacionales a nivel algorítmico de las personas (Stanovich y West, 2000).

En paralelo al estudio de la vinculación entre la eficacia en el razonamiento analítico y medidas de inteligencia general, científicos cognitivos han investigado dos estrechos correlatos: la memoria de trabajo y la edad de desarrollo (Evans, 2008). La capacidad de memoria de trabajo es bien conocida por predecir niveles de rendimiento en una amplia gama de tareas cognitivas, y por poseer un vínculo directo con la teoría de doble procesamiento (Barrett, Tugade y Engler, 2004). Por su parte, los estudios ligados al desarrollo evolutivo también han sido reveladores. Un claro exponente de ello ha sido el hallazgo de ciertas habilidades de pensamiento analítico que se desarrollan con la edad y serían favorecedoras del rendimiento en pruebas de inteligencia general. Pese a ello, recientemente se ha afirmado que sería la habilidad, y no la edad, el predictor más importante en el razonamiento analítico (Evans, 2008).

No obstante lo afirmado hasta aquí, existen algunas tareas en la literatura heurística y de sesgos que al parecer carecerían de cualquier forma de asociación con la capacidad cognitiva (Stanovich y West 2000). Es decir, pensar de forma racional significa adoptar metas apropiadas, tomar medidas adecuadas dadas las metas y creencias de uno, y tener creencias acordes con la evidencia disponible (Stanovich, West y Toplak, 2013). De este modo, si bien las pruebas de inteligencia medirían muchos de los aspectos considerados importantes en torno al pensamiento, no es posible decir, sin embargo, que evalúen completamente su grado de racionalidad. Así, la inteligencia estaría muy ligada a ciertos aspectos del pensamiento racional pero, débil o nulamente, vinculada a otros (Stanovich et al., 2013).

En este punto, adquieren especial relevancia las comunicaciones de Stanovich y West (2000) y Stanovich (2012), que, a través de una gran variedad de tareas de la literatura heurística, declaran haber encontrado ciertas disposiciones de pensamiento capaces de predecir la varianza entre los modelos normativos y descriptivos, una vez que los efectos de la inteligencia general han sido controlados. De este modo, frente al primer enfoque, surgen nuevas propuestas desde las que se señala que, para entender los procesos que subyacen al razonamiento humano, es necesario tener en cuenta variaciones tanto a nivel algorítmico, como a nivel intencional (Seoane et al., 2007).

En un intento por identificar las disposiciones de pensamiento mencionadas, Stanovich (2012) propone la siguiente lista: comprensión epistemológica, voluntad de cambiar de perspectiva, voluntad de descontextualizar, disposición a considerar alternativas, apertura activa de pensamiento, necesidad de cognición, y necesidad de cierre. Según este autor, dichas disposiciones reflejarían ciertos tipos de preferencias 
cognitivas, como, por ejemplo, la tendencia a recopilar información antes de arribar a un resultado, la disposición a pensar ampliamente acerca de un problema antes de resolverlo, o la tendencia a pensar en las consecuencias futuras antes de actuar (Stanovich y West, 1998). En consecuencia, las disposiciones de pensamiento serían consideradas como características psicológicas sustentadoras del pensamiento y la acción racional (Stanovich, 2012).

Por su parte, Sternberg et al. (2008) definen los estilos o disposiciones como las variaciones individuales en los enfoques de las tareas que pueden hacer una diferencia en la forma y eficacia con que una persona percibe, aprende o piensa. Es decir, para estos autores dichas disposiciones no serían una habilidad en sí, sino más bien, el modo en que uno se inclina a usar sus propias habilidades. Asimismo, cuando hablan de diferencias individuales en estilos de pensamiento, lo hacen en términos de variaciones entre dichos estilos, pero sin referirse a lo que es mejor o peor entre ellos. De este modo, ningún estilo sería bueno o malo en sí, sino más bien materia de ajuste entre la persona y la tarea en cuestión. Por último, ofrecen una lista de ciertas características que compartirían estas disposiciones. Así, explican que las personas pueden diferir en el nivel de fuerza con que se aferran a un estilo, así como en el grado de flexibilidad con que utilizan los mismos. De este modo, mientras algunas personas tendrían fuertes inclinaciones por algunos estilos, otras tendrían preferencias más bien débiles. Asimismo, mientras algunas podrían alternar fácilmente entre estos, otras no podrían hacerlo. Finalmente, señalan que las disposiciones no solo tendrían la propiedad de socializarse, sino que, además, podrían sufrir variaciones a lo largo de la vida.

Al realizar una síntesis de lo hasta aquí expuesto, es posible decir que en el estudio de diferencias individuales en razonamiento analítico, dos han sido los niveles de análisis principalmente desarrollados: El nivel algorítmico o de capacidades cognitivas, y el nivel intencional o de disposiciones de pensamiento. El primero de estos niveles referiría al tipo de proceso cognitivo relacionado con la base cognitiva de las pruebas de inteligencia. La velocidad de percepción, la precisión al discriminar la información, la capacidad de memoria de trabajo y la eficiencia en la recuperación de la información almacenada, serían ejemplos de estas capacidades. Según la concepción de Baron (1985), dichas capacidades no podrían mejorarse a corto plazo por efecto de la instrucción; sin embargo, podrían ser afectadas por la práctica a largo plazo. Un análisis más exhaustivo de esta propuesta puede encontrarse en Baron (1991).

En contraste con el nivel algorítmico, el nivel intencional se distinguiría por ser de condición más maleable. En este sentido, Baron (1985) explica que aunque no es posible mejorar la memoria de trabajo por medio de la instrucción, es posible en cambio decirle a una persona que invierta más tiempo en la resolución de un problema, antes de resignar su solución. Así, las disposiciones de pensamiento serían aquellas estructuras reflexivas capaces de servir como mecanismos explicativos de las diferencias a un nivel de análisis intencional, vinculadas con objetivos y valores de las personas e indicadoras de tendencias de autorregulación pragmática (Stanovich, 2012).

Desarrollados ambos niveles de análisis, se comprende cómo las diferencias individuales en razonamiento deductivo pueden surgir de una amplia variedad de factores cognitivos (Stanovich y West 1998, Seoane et al., 2007). Dichos factores, si bien en ocasiones pueden no actuar de manera completamente predecible, lejos se encuentran de pertenecer a un grupo de variables meramente azarosas (Stanovich, 2012).

En la actualidad, aunque se sostiene que la racionalidad es un constructo más amplio que la inteligencia, se considera que esta última forma parte de la primera (Stanovich, 2012; Burgoon, Henderson y Markman, 2013). Tal como ha sido remarcado, para ser racional, una persona no solo debe tener sus creencias bien calibradas, sino además, debe actuar de manera alineada con estas. Ambas propiedades pertenecen al nivel intencional. Al mismo tiempo, la persona debe contar con una maquinaria de nivel algorítmico adecuada. Es decir, tener las habilidades necesarias que le permitan llevar a cabo sus metas, procesando el medio ambiente de manera tal que el desarrollo de creencias adecuadas derive en la elección del mejor curso de acción (Stanovich, 2012). Así, las diferencias en el pensamiento racional podrán surgir tanto a causa de diferencias a nivel algorítmico (inteligencia), como a nivel intencional (disposiciones de pensamiento).

Debido a la creencia según la cual las disposiciones de pensamiento y la capacidad cognitiva difieren en su grado de maleabilidad, será importante para investigaciones futuras determinar la proporción relativa de influencia que cada nivel posee sobre el pensamiento racional (Stanovich y West, 1997). Este último punto reviste especial interés, dado que 
en la medida que mayor sea la proporción de influencia atribuible a las disposiciones de pensamiento, mayor serán las posibilidades de mejorar el pensamiento crítico por medio de la instrucción y, en consecuencia, de disminuir las diferencias entre individuos (Baron, 1985).

\section{Otras variables de interés en el estudio de diferencias individuales: desarrollo, género y cultura}

\section{Desarrollo}

Muchos son los interrogantes que se han disparado en torno al desarrollo y la manera en que este es capaz de condicionar el razonamiento humano. Pese a ello, dos focos de interés principal resultan fácilmente distinguibles. Por un lado, los psicólogos cognitivos han centrado su atención en el estudio de las diferencias que se manifiestan a temprana edad, es decir, el período durante el cual la capacidad de razonamiento comienza a florecer. Por el otro, algunos teóricos han detenido su observación en las diferencias que aparecen durante la adultez mayor, período en el que dichas capacidades comienzan a declinar.

En relación con los estudios de razonamiento realizado con niños, uno de los fenómenos que mayor atención ha recibido es el relacionado con la atribución de falsas creencias en terceros. Razonar sobre lo que otras personas creen resulta esencial para la interpretación de la acción humana. Pese a ello, una extensa cantidad de literatura científica ha detectado cierta dificultad por parte de los pequeños a la hora de evaluar creencias ajenas, sobre todo cuando estas difieren del propio punto de vista o refieren a contenidos falsos (Birch y Bloom, 2003).

Una de las líneas más fuertes de investigación plantea que dicha limitación podría ser explicada por un fenómeno al que denominan maldición del conocimiento (Birch y Bloom, 2007). Dicho fenómeno consistiría en la tendencia a dejarse influenciar por el propio conocimiento, al momento de juzgar los conocimientos de una tercera persona más ingenua o ignorante que uno mismo. Según estos autores, se trata de una propensión encontrada también en adultos, pero que en los pequeños se expresaría de forma exagerada. Asimismo, explican que esta tendería a manifestarse sobre todo en forma asimétrica, estando limitada a los casos en los que el niño posee mayores conocimientos que la persona a la que evalúa. Por el contrario, cuando el menor es más ignorante que la persona evaluada, dicho sesgo no se produciría, confirmando de este modo la hipótesis enunciada. Finalmente, los autores plantean que el sesgo no excluiría, sin embargo, otras explicaciones, y que bien podría darse el caso de un condicionamiento mixto entre este sesgo y otras limitaciones de tipo conceptuales o de procesamiento.

Un segundo fenómeno hallado en la niñez proviene del área del razonamiento relacional, dentro del cual también ha sido demostrada cierta complejidad por parte de los menores al momento de realizar este tipo de tareas. En relación con ello, ciertas investigaciones señalan cómo las palabras y el lenguaje ayudarían a los niños a comprender y hacer uso de relaciones en diferentes niveles. Rattermann, Gentner y DeLoache (1990), por ejemplo, han demostrado que etiquetar una serie de objetos que variaban en su tamaño (como papá, mamá y bebé) ayudaban a los preescolares a razonar acerca de las relaciones de tamaño. También Herbert y Heyne (2000) resaltan la importancia del lenguaje en el desarrollo del razonamiento inferencial. Según estos autores, las palabras no solo permitirían que el niño relacione nuevas tareas con estructuras relacionales conocidas, sino que además posibilitarían la conexión con ideas previamente adquiridas, y facilitarían el paso de representaciones más concretas a otras de carácter más abstracto.

Además de las palabras, otros contextos demostraron ser facilitadores de tareas relacionales en pequeños. Tal es el caso de ciertas situaciones o eventos familiares, como el ir al cine o salir de compras a un supermercado (Son, Smith, Goldstone y Leslie, 2012). Los desarrollos en esta línea sugieren que el razonamiento relacional derivaría de ciertas representaciones, como esquemas de eventos (Schank y Abelson, 1995). Por ejemplo, el ir de compras a un supermercado tendría una estructura común que se capturaría en el esquema salir de compras, a través de una variedad de experiencias específicas de ir al supermercado (Son et al., 2012). Dichos esquemas podrían definirse como entidades cognitivas, de carácter abstracto, capaces de ordenar y estructurar emociones, percepciones y experiencias. Asimismo, estos esquemas se organizarían en espacios ocupados con diferentes unidades de conocimiento, las cuales resultan necesarias para dar respuesta a situaciones estructuralmente similares (Schank y Abelson, 1995).

Siguiendo la hipótesis propuesta, cabría esperar que la evocación de una estructura-evento familiar resultase de mayor utilidad en la promoción de 
interpretaciones relacionales, que una menos conocida. Pese a ello, la evidencia parece demostrar que si los niños evocan un recuerdo demasiado específico en lugar de una historia-esquema más general, podrían tener dificultades al momento de aplicar dicho esquema al presente problema relacional. Es decir, para que los niños puedan beneficiarse de la utilización de esquemas en tareas relacionales, estos deben saber lo suficiente acerca de la estructura de eventos como para hacer uso inferencial al respecto. Sin embargo, no deben contar con demasiada especificidad en el delineamiento de la misma, dado que ello dificultaría la aplicación relacional a nuevos problemas (Brown, Kane y Echols, 1986). En convergencia con este punto, investigaciones provenientes del área de la atención explican que cuando el foco de la misma ha sido dirigido hacia alguna característica concreta, resulta extremadamente difícil para los niños superar esta inercia atencional si les es solicitado el cambio a otra diferente (Kirkham, Cruess y Diamond, 2003). Finalmente, si bien este fenómeno no sería en absoluto exclusivo de un estadio temprano (confundir detalles concretos y relaciones abstractas hace difícil el razonamiento relacional incluso para algunos adultos), su fuerza y frecuencia serían mayores en los niños más pequeños (Son et al., 2012).

Dirigiendo ahora la mirada hacia el segundo eje de interés, esto es, el de adultos mayores, se reconoce una tonalidad diferente en torno a sus hallazgos. En este sentido, los estudios han centrado su observación en los cambios y pérdidas que acontecen con la edad, más que en la documentación de destrezas adquiridas. Así, la asociación entre una marcada declinación en la memoria de trabajo y procesos de aprendizaje, y el incremento de la edad, ha quedado bien establecida a lo largo de una extensa evidencia empírica. Wild-Wall, Falkenstein y Gajewski (2011), por ejemplo, explican que una degradación del sustrato neural relacionada con la edad habría sido encontrada en el lóbulo frontal, lo cual puede estar relacionado con un déficit observado en la memoria de trabajo. También Peters, Hess, Västfäll y Auman (2007) denuncian diferencias en adultos mayores vinculadas al procesamiento deliberativo y afectivo (en apartados anteriores, sistema analítico y heurístico respectivamente). Sin embargo, señalan que el deterioro en el primer sistema sería innegable con la edad, al tiempo que los cambios en el segundo serían apenas perceptibles.

Una hipótesis ampliamente aceptada en torno a este fenómeno es el hallazgo de una creciente difi- cultad a edades avanzadas para filtrar información irrelevante en el procesamiento de datos. Dicha dificultad llevaría a sobrecargar los recursos de la memoria de trabajo y, en consecuencia, a reducir su rendimiento (Wild-Wall et al., 2011). Este debilitamiento, asimismo, fomentaría la utilización de formas más implícitas de procesamiento. De este modo, los adultos podrían volverse más expertos en su uso $y$, en consecuencia, tender a confiar más plenamente en el mismo (Peter et al., 2007).

Matsuyoshi, Osaka y Osaka (2014) encontraron también una disminución en la capacidad de la memoria de trabajo visual, tanto en adultos jóvenes, como en los de mayor edad. Sin embargo, refirieron que los del primer grupo eran capaces de superar más rápido esta dificultad si eran provistos de un tiempo adicional para terminar la tarea. Por último, WildWall et al. (2011) hallaron un decaimiento en el rendimiento cognitivo tanto en la mediana, como en la tercera edad. Sin embargo, comprobaron que los participantes más jóvenes eran capaces de ajustarse con mayor flexibilidad a la resolución de tareas en aquellos momentos en que la memoria de trabajo se encontraba sobrecargada.

Pese a la evidencia poco optimista presentada hasta aquí, existen, no obstante, ciertos estudios con mayor esperanza al respecto. William, Dunlop y Abdi (2012), por ejemplo, se interesan por los cambios vinculados a la comprensión del lenguaje. En sus estudios, estos autores manifiestan que algunos adultos mayores serían capaces de analizar información semántica y comprender representaciones lingüísticas complejas tan bien, o incluso mejor que adultos más jóvenes, en aquellos contextos en los que dicha comprensión no depende de la memoria operativa. Asimismo, señalan que la capacidad para generar inferencias globales permanecería estable a lo largo de la edad, al no encontrar diferencias significativas entre los jóvenes de edad universitaria, los adultos de mediana edad y los de edad avanzada. Finalmente, concluyen que los cambios en habilidades verbales, en particular, mostrarían un patrón de aparición más tardío, así como una mayor variabilidad en su edad de aparición y su tasa global de cambio.

En trabajos relacionados, Nyberg et al. (2003) declaran de manera optimista que no todas las formas de memoria se ven igualmente afectadas por el paso de tiempo. Así, mientras la memoria episódica y la memoria de trabajo serían consideradas dos de los mayores exponentes tendientes al deterioro, la memoria primaria o la memoria procedimental habrían 
de sufrir pocos cambios con la edad, al tiempo que la memoria semántica presentaría gradientes positivos.

Por último, Nyberg, Lövde'n, Riklund, Lindenberger y Bäckman (2012) ofrecen evidencia respecto de una marcada heterogeneidad en el envejecimiento cerebral. Así, mientras algunas personas manifestarían un visible deterioro cognitivo a muy temprana edad, ciertos subgrupos de adultos mayores mostrarían poco o ningún cambio cerebral, junto con un rendimiento cognitivo intacto. Según estos autores, la evidencia estaría señalando diferencias sustanciales en el rendimiento cognitivo durante la tercera edad. Estas se materializarían tanto en la naturaleza, como en el grado del cambio efectuado, dejando incluso abierta la posibilidad a ciertos casos en los que dicho rendimiento se mantendría inalterable.

Pese a que los interrogantes en torno al proceso de envejecimiento todavía son numerosos, los autores explican que estas diferencias serían el resultado de la interacción tanto de los genes entre sí, como de los genes con el contexto. Es decir, además de los determinantes genéticos señalados, los factores ambientales y ciertas opciones de estilo de vida desempeñarían un papel fundamental en el mantenimiento cerebral y el consecuente rendimiento cognitivo durante la vejez (Nyberg et al., 2012).

\section{Género}

No sin especial intención ha sido dejado para último lugar la discusión en torno de las diferencias vinculadas al género y la cultura de las personas. Tal como sucede en aquellas áreas en las que entran en juego diferencias entre grupos demográficos, se trata aquí de hallazgos tan interesantes como controvertidos. Lamentablemente, al entrar en este terreno, gran parte de la literatura científica se carga emocionalmente, resultando a menudo en conclusiones poco claras o ambivalentes (Johnson, Carothers y Deary, 2008).

Tomando en primer lugar los desarrollos ligados al género, muchas de sus investigaciones han estado direccionadas por el interrogante de si las diferencias en habilidades cognitivas entre hombres y mujeres verdaderamente existen y, en tal caso, en qué momento del desarrollo se vuelven evidentes (Halpern et al., 2007). Otras líneas han dirigido su atención con respecto al grado en el que estas diferencias serían explicadas por factores innatos, o por el contrario, atribuibles a características del contexto (Dekker, Krabbendam, Aukje, deGroot y Jolles, 2013). Estos últimos interrogantes explicarían el hecho de que muchas de las investigaciones vinculadas a diferencias de género hayan tenido lugar muy temprano en el desarrollo. Dado que los recién nacidos han tenido menor tiempo de interacción con el ambiente, se cree que las diferencias encontradas en este punto serían, presumiblemente, atribuibles a un origen biológico (Halpern et al., 2007). Sin embargo, este supuesto no sería del todo compatible con la literatura biológica, la cual establece que para muchas especies las diferencias de género no serían evidentes en la infancia, sino por el contrario, harían su aparición más bien tarde en el desarrollo (Halpern et al., 2007).

Según Halpern (2004), dentro del campo de las habilidades cognitivas, resulta extremadamente difícil hablar de influencias biológicas e influencias ambientales como dominios separados, dado que estas continuamente se encuentran interactuando y modificándose entre sí. En consonancia con ello, Johnson et al. (2008) señalan que la cuestión de diferencias de género en procesamiento cognitivo conforma un conjunto de preguntas enmarañadas, dado que su análisis dependería de la evaluación de múltiples factores, tales como el tipo de habilidades que están bajo estudio, la manera en que se evalúan dichas habilidades y el momento evolutivo examinado, entre otros.

Considerando, por ejemplo, el aspecto del contenido, tres han sido las habilidades cognitivas fundamentalmente estudiadas: habilidades verbales, visuoespaciales y cuantitativas (Halpern et al., 2007). Derivado del postulado de inteligencias múltiples (Gardner, 2001), pareciera haber un acuerdo general según el cual los hombres tenderían a obtener mejores resultados en ciertos tipos de pruebas, mientras las mujeres los superarían en otras diferentes (Johnson et al., 2008). Numerosas investigaciones respaldan estos hallazgos. Uno de los primeros meta-análisis realizados dentro del área (Maccoby y Jacklin, 1974) ha encontrado que a partir de la adolescencia las mujeres tenderían a desenvolverse mejor en pruebas de tipo verbal, mientras los varones obtendrían mejores resultados en pruebas de tipo cuantitativas o visuoespaciales. En un estudio más reciente, Dekker et al. (2013) encontraron que las niñas tendían a superar a los muchachos en velocidad de procesamiento en tareas que involucraban números y alfabetos, mientras los niños eran más rápidos en tareas relacionadas con medidas simples. Otros estudios, en cambio, resaltan la ventaja de los varones al momento de evaluar el razonamiento matemático. Mills, Ablard y Stumpf (1993) presentaron evidencia respecto de diferencias de género en razonamiento 
matemático ya desde el segundo grado de nivel primario (entre alumnos superdotados intelectualmente), al tiempo que Robinson, Abbott, Berninger y Busse (1996) informan de su existencia aun antes del jardín de infantes.

Pese a todas las diferencias encontradas, para Halpern et al. (2007) una pregunta importante que debe realizarse es el grado en el que las distintas razas, grupos étnicos y pueblos de todo el mundo muestran el mismo patrón de resultados. De este modo, aunque las diferencias de género en habilidades cognitivas sea una cuestión muy compleja, para estos autores el hecho de que existan algunas pruebas en las que las mujeres en todos los grupos raciales y étnicos tiendan a sobresalir, y algunas tareas en las que los hombres en todos los grupos tiendan a predominar, hace que resulte verdaderamente difícil para cualquier teoría única, tales como la motivación, el aprendizaje, o la biología, explicar dicha diferencia.

Considerando, por otro lado, el momento evolutivo en el cual estas evaluaciones son llevadas adelante, nuevos matices entran en escena. Según Spelke (2005), numerosos estudios coinciden en afirmar que los desarrollos cognitivos relacionados con el razonamiento cuantitativo y el conocimiento de objetos se desarrollarían igualmente bien en niños y niñas durante la primera infancia. Asimismo, agrega que por lo general se requerirían muestras muy grandes para detectar diferencias durante los primeros años de vida. Por otro lado, en un estudio a gran escala realizado por Camarata y Woodcock (2006), se ha demostrado que la magnitud de las diferencias de género en la velocidad de procesamiento estaría altamente influenciada por el rango de edad observado. Según los resultados obtenidos por estos autores, la ventaja de las niñas sería relativamente menor en niños pequeños (nueve años y más jóvenes), mayor en adolescentes jóvenes (10-13 años de edad), y aún más grande en adolescentes mayores (14-18 años de edad). Sin embargo, la misma desaparecería casi por completo en estudiantes universitarios y adultos jóvenes (17-34 años de edad). Respaldando la evidencia anterior, estudios de neuroimagen demostraron que los cambios en el desarrollo durante la adolescencia serían diferentes para varones y mujeres, al existir un relativo retraso en el desarrollo del cerebro del varón respecto del de la mujer (Dekker et al., 2013). Al parecer, el volumen total del cerebro alcanzaría su pico máximo más tarde en los varones (14,5 años), que en las mujeres (10,5 años). Esto estaría sugiriendo que, junto con la edad, el género sería un posible factor influyente en la velocidad de procesamiento, al menos en torno a ese período del desarrollo (Dekker et al., 2013). En congruencia con estos hallazgos, otros estudios habrían manifestado diferencias tanto a nivel estructural como funcional, en áreas del cerebro involucradas a funciones cognitivas (Clements et al., 2006). Así, un mayor volumen de materia blanca en los varones estaría asociado a un mejor rendimiento espacial, mientras una mayor simetría bilateral en las mujeres estaría asociada con un mejor procesamiento del lenguaje.

Finalmente, Halpern et al. (2007) resumen la complejidad del fenómeno de género explicando que, en definitiva, habría ciertas diferencias entre los cerebros masculinos y femeninos que serían incentivadas desde un inicio por las hormonas prenatales, algunas otras que serían estimuladas por la acción de las hormonas que aparecen más tarde en el desarrollo, y algunas más que serían atribuibles a las diferentes experiencias de vida, incluyendo la del ambiente prenatal.

Tal como fue enunciado desde un principio, la cuestión de diferencias de género en habilidades cognitivas continúa atrapada en el debate entre el determinismo biológico y el condicionamiento ambiental. Lamentablemente, no existe un factor único capaz de explicar por sí mismo todas las diferencias encontradas. Pese a ello, la evidencia general parece dejar en claro que ciertas diferencias de género existen, y serían, de hecho, factibles de corroborar. Una revisión de la evidencia recolectada, pertinente para esta discusión, se presenta en Colom, Espinosa, Abad y García (2000), y Dekker et al. (2013).

\section{Cultura}

Por último, se aborda la cuestión de la cultura, eje tanto o más controvertido que el anterior. Diversas posturas coexisten en relación con la temática: desde aquellos que proponen que las diferencias en inteligencia entre razas y etnias se deben exclusivamente a factores genéticos (Rushton y Jensen, 2005); pasando por los que plantean que dichas diferencias son en realidad un mero producto de la interacción social (Sowell, 2005); llegando incluso hasta los que proponen que estas no existen, tratándose únicamente de una transferencia inapropiada de ciertas pruebas $y$ test a diferentes culturas (Sternberg, Grigorenko, y Kidd, 2005).

Bender y Beller (2011) manifiestan que el estudio intercultural es algo no solo posible, sino deseable, si 
lo que se quiere es explorar la diversidad cognitiva. Sin embargo, consideran que se trata de una problemática sumamente compleja. Algunas de las dificultades que aparecen en escena surgen de lo que Medin, Bennis y Chandler (2010) dieron en llamar las desventajas propias del campo. Una de estas desventajas consistiría en la tendencia a dejar a una de las culturas comparadas sin marcar, convirtiéndola así en el estándar respecto del cual las otras se desviarían. Como consecuencia de esto, las peculiaridades de la cultura sin marcar terminarían siendo puntos ciegos culturales. Otra de las desventajas consistiría en la tendencia a considerar otras culturas (y en ocasiones conjuntos enteros de culturas) como más homogéneas que la propia, y en consecuencia como más homogéneas de lo que realmente son. Esto resulta evidente, por ejemplo, cuando todos los nativos americanos son equiparados a los indios, o cuando a chinos, japoneses y surcoreanos se les conoce como los asiáticos. Finalmente, una tercera desventaja estaría dada por la existencia de una confianza excesiva en la equivalencia de las tareas a través de las culturas. Esto estaría suponiendo al menos dos preocupaciones: por un lado, la preocupación por cómo se entienden estas tareas y cómo habrían de ser interpretados en diferentes contextos culturales; por el otro, la preocupación respecto de qué revelarían finalmente los datos obtenidos (Bender y Beller, 2011). En relación con esto, Hunt y Carlson (2007) agregan que las puntuaciones de las pruebas muchas veces son tratadas como indicadores infalibles de la capacidad cognitiva, al ser interpretadas como una característica invariable del sujeto. Lo que muchos investigadores suelen olvidar, sin embargo, es que el subconjunto de habilidades evaluadas viene finalmente determinado por una decisión social, por lo que esta considera que es importante, y por el propósito de la prueba en sí. Una revisión más extensa sobre este problema puede hallarse en Burgoon et al. (2013).

Las desventajas propias del campo serían entonces el resultado de presunciones que los investigadores tendrían sobre su campo y sobre las reglas del juego. El problema residiría en que tales presunciones actuarían aumentando la distancia psicológica entre los investigadores y los sujetos de la investigación, y por tanto, dificultando la toma de perspectiva (Bender y Beller, 2011). Pese a lo enunciado, Medin et al. (2010) afirman que dichas desventajas podrían ser controladas. Para ello, sin embargo, se requeriría un esfuerzo constante en el marcado del grupo sin marcar, la colaboración con el grupo investigado, la realización de investigaciones sobre los términos de la cultura respectiva, y la toma obligada de múltiples perspectivas.

Mencionadas las anteriores limitaciones, es posible continuar con ciertos hallazgos dentro del área. En términos generales, estos refieren a características más bien vagas que a resultados determinantes. Knight y Nisbett (2007), por ejemplo, han encontrado que los asiáticos estarían inclinados a razonar y percibir de una manera más holística, a hacer hincapié en relaciones y similitudes entre objetos y eventos, y a deslizarse en un amplio campo perceptual. Los occidentales, en cambio, tenderían a razonar de forma más analítica, a focalizar en un objeto central y a razonar utilizando reglas y categorías. Los autores explican que estas tendencias estarían arraigadas en las diferentes prácticas sociales de cada región. En este sentido, se sabe que los asiáticos del este son más dependientes entre sí, y tienden a centrar su atención en el mundo social y el campo como un todo. Por su parte, los occidentales suelen ser menos dependientes, y tendientes a focalizar su atención en un objeto único (Knight y Nisbett, 2007).

Hasta el momento, el estudio de la variabilidad cognitiva derivada de diferencias culturales se ha limitado principalmente a comparaciones entre países. Sin embargo, dado que las naciones son productos recientes de complejos procesos históricos, no es posible decir que pertenezcan a entidades completamente homogéneas (Knight y Nisbett, 2007). Así, no sería raro encontrar diferencias entre algunas subculturas de una misma nación. Estos autores, asimismo, apuntan que las comparaciones intra-nacionales tampoco deberían limitar su estudio a diferentes regiones, dado que las diferentes clases sociales también pueden diferir en sus prácticas. Comparaciones entre diferentes niveles sociales en Estados Unidos, por ejemplo, demostraron que diferentes interacciones entre padres e hijos influirían en la forma en que los niños adquieren el conocimiento (Andersen, 1986). Por su parte, Allan (1979) declara que a lo largo del mundo ha encontrado que individuos que pertenecían a clases sociales menos favorecidas tenían un mayor contacto con su familia, se percibían con una menor autoeficacia y reportaban un menor locus de control interno. Esto quizás relacionado al hecho de que efectivamente solían tener menos oportunidades de elección y autodirección en sus vidas. La socialización de niños de clases más favorecidas, en cambio, estaba relacionada con una enseñanza más independiente, mayor posibilidad de autodescubrimiento y mayor tendencia a 
enfatizar en la obediencia (Knight y Nisbett, 2007). Si esto es así, cabría esperar que los individuos pertenecientes a clases menos favorecidas sean relativamente más interdependientes (dado que sus prácticas sociales estarían asociadas a un mayor sentido de pertenencia con los demás miembros de su entorno), y tengan una orientación más holística en sus hábitos cognitivos (Knight y Nisbett, 2007).

Como ha sido expuesto, diversas líneas de investigación intentan dar cuenta del fenómeno cultural. Desde un punto de vista constructivista, por ejemplo, se postula que las culturas son tradiciones de pensamiento y práctica, y que formar parte de una implica impregnarse de una gran cantidad de representaciones culturales (esquemas, categorías, procedimientos, metas) conscientes e inconscientes (Weber y Morris, 2010). Desde este modelo, las representaciones guiarían las sentencias y resoluciones de problemas, al tiempo que restringirían la atención, la búsqueda de evidencia y el proceso de inferencia. Asimismo, la activación de estas representaciones estaría relacionado con su aplicabilidad y accesibilidad, lo que a su vez dependería de cuán reciente ha sido la experiencia y de la frecuencia de su uso (Weber y Morris, 2010).

Para estos autores, la noción de que las representaciones no son algo que permanezca siempre activo, permitiría a su vez la comprensión de cómo los individuos policulturales son capaces de desenvolverse en más de una cultura sin mezclar sus sesgos o prejuicios. Asimismo, la premisa de que las representaciones son dinámicas, posibilitaría a la psicología cultural apartarse de algunos supuestos simplificadores o estereotípicos.

Desde una orientación más biológica, estudios con gemelos monocigóticos (idénticos) y dicigóticos (fraternos) han intentado desentrañar en qué medida características fenotípicas complejas (como el rendimiento de la memoria, la personalidad...) serían el producto directo de los genes, del medio ambiente compartido o de influencias ambientales no compartidas (King y Suzman, 2008). Dentro de este marco, algunos genetistas conductuales demostraron que muchos rasgos complejos (como aquellos ligados a la cognición) serían resultado de una heredabilidad sustancial (aproximadamente el 50\% de la variación sería explicable genéticamente), atribuyendo el porcentaje restante a efectos ambientales de diversa índole (Finkel, Reynolds, McArdle y Pedersen, 2005). Estos datos estarían apoyando la concepción de que el relativo enriquecimiento del medio ambiente de un gemelo podría causar divergencias en la trayectoria de su cognición. Sin embargo, estas observaciones seguirían dejando abierta la pregunta respecto de qué aspectos del medio ambiente serían los responsables de dichas influencias (King y Suzman, 2008).

Por último, desde una posición ciertamente moderada, Hunt y Carlson (2007) alertan sobre la conveniencia de ajustar dentro del campo las expectativas a los resultados encontrados. Para estos autores, si hubiera alguna característica única dentro del área capaz de explicar las discrepancias entre los diversos grupos, esta sin duda habría sido encontrada. Señalan, sin embargo, que es probable que la discrepancia entre diferentes culturas se deba a múltiples, pequeños y sutiles efectos sociales, muchos de los cuales están relacionados con las prácticas de los grupos en cuestión (actitudes hacia la educación, efectos indirectos de las prácticas de salud, grados relativos de solidaridad familiar, etc.). Si bien la suma de estos pequeños efectos podría ser causal de un gran efecto, la tarea de identificar y hacer frente a una multitud de pequeñas causas sería sin duda mucho más compleja. En cualquier caso concluyen que, si en verdad una variedad de pequeñas causas son las que se encuentran covariando con la capacidad cognitiva, pretender dar cuenta de dicha variación desde un único modelo explicativo sería una empresa sumamente ambiciosa (Hunt y Carlson, 2007).

\section{Discusión}

A lo largo de este artículo, ha sido abordada la cuestión de diferencias individuales en razonamiento deductivo. Para el tratamiento de la temática, se han considerado dos de las principales teorías vinculadas al campo: por un lado, la Teoría de Modelos Mentales (Johnson-Laird, 1983; Johnson-Laird y Byrne, 1991, citado por Johnson-Laird, 2010). Por el otro, la Teoría de Doble Procesamiento (Stanovich y West, 1997, 2000; Evans, 2003). La ммт postula que el razonamiento es un fenómeno semántica y sintácticamente impulsado, en lugar de un proceso basado en reglas lógicas, y que los sujetos razonan manipulando modelos mentales. Estos serían representaciones semánticas de objetos y situaciones del mundo real, elaboradas por los sujetos a partir de la información descrita en las premisas, así como también de su propio conocimiento del mundo (Johnson-Laird, 2006). Las teorías ubicadas dentro del marco del doble procesamiento, en cambio, coinciden en plantear dos sistemas diferentes para explicar el razonamiento humano en 
lugar de un único mecanismo universal de inferencia. Así, dentro de estas teorías se asume cierto consenso a la hora de hablar de un sistema 1, o sistema heurístico, caracterizado como intuitivo, rápido y de bajo consumo atencional; en co-existencia con un sistema 2, o analítico, descripto como reflexivo, lento $\mathrm{y}$ de alto consumo atencional (Schroyens et al., 2000).

En este contexto, el propósito de la presente contribución fue realizar una revisión narrativa de aquellos trabajos que abordan la temática bajo estudio. Para ello, fueron relevados aportes clásicos (Stanovich y West, 2000) y algunos recientes (Avery et al, 2013; Restrepo 2015). Inicialmente se trataron las variables relacionadas con las habilidades cognitivas, y la disposición al conocimiento. Posteriormente, se analizaron aquellas otras vinculadas con el desarrollo, el género y la cultura de las personas.

En coherencia con lo argumentado desde cada una de estas áreas, se concluye que el estudio de diferencias individuales en cognición y razonamiento deductivo pertenece a un terreno sumamente complejo, atravesado por una multitud de factores intervinientes. Las experiencias tempranas, las limitaciones biológicas, la política educativa y el contexto cultural serían solo algunos de los factores en juego (Halpern et al., 2007).

Embarcar una empresa de síntesis exhaustiva sobre esta problemática escapa sin dudas el alcance de este trabajo. Se considera, sin embargo, que esfuerzos tendientes en esta línea podrían contribuir a una mejor comprensión del campo. Se sugiere para próximas investigaciones comenzar por identificar puntos comunes entre las diferentes teorías, a fin de delinear una base común de partida. Se propone asimismo la consideración de otras variables relevantes en razonamiento, como es el caso de la experticia y rasgos de la personalidad, excluidas en esta oportunidad por razones de espacio. En relación con este punto, si bien la evidencia general sugiere cierta vinculación entre factores cognitivos y rasgos de la personalidad (Restrepo, 2015), se considera que el desarrollo de una línea que profundice con relación a estos y las diferencias en razonamiento, sería del todo interesante. Finalmente, tal como fue expuesto, el trabajo ha centrado su atención en dos de las principales teorías dentro del campo. A pesar de existir otras teorías ligadas a razonamiento, como es el caso de la psicología de la prueba planteada por Rips (1989; 1994), no fueron incluidas en esta oportunidad por no encontrarse dentro de las mismas un tratamiento específico en torno a la temática. Con este último punto, empero, no se pretende cerrar el análisis a las teorías exclusivamente tratadas. Por el contrario, se deja abierta la posibilidad a que futuros trabajos puedan contribuir desde diferentes enfoques. La condición narrativa del presente artículo puede considerarse una limitación en cuanto a su capacidad explicativa, dado que para decidir sobre el valor de la evidencia serían necesarios nuevos estudios que combinen los resultados de las diversas investigaciones realizadas, para cada una de las variables propuestas. Sin embargo, contribuciones de esta naturaleza podrían realizarse por medio de futuros trabajos metanalíticos (Schmidt, 1996; Grissom y Kim, 2005; 2012).

\section{Referencias}

Allan, G. A. (1979). A sociology of friendship and kinship. London: Allen \& Unwin.

Andersen, E. S. (1986). The acquisition of register variation by Anglo-American children. En B. Schieffelin y E. Ochs (Eds.), Language socialization across cultures (pp. 153-161). Cambridge: Cambridge University Press.

Avery, R. E., Smillie, L. D. y de Fockert, J. W. (2013). The role of working memory in achievement goal pursuit. Acta Psychologica, 144, 361-372. doi: 10.1016/j. actpsy.2013.07.012

Barlow, D. H. y Nock, M. K. (2009). Why can't we be more idiographic in our research? Perspectives on Psychological Science, 4(1), 19-20. doi: 10.1111/j.17456924.2009.01088.x

Baron, J. (1985). Rationality and intelligence. Cambridge: Cambridge University Press.

Baron, J. (1991). Beliefs about thinking. En J. F. Voss, D. N. Perkins y J. W. Segal (Eds.), Informal reasoning and education (pp.169-186). Hillslade, NJ: Lawrence Erlbaum Associates.

Baron, J. (2008). Thinking and deciding. New York: Cambridge University Press.

Barrett, L. F., Tugade, M. M. y Engle, R. W. (2004). Individual differences in working memory capacity and dual-process theories of the mind. Psychological Bulletin, 130(4), 553-573. doi: 10.1037/0033-2909.130.4.553

Bender, A. y Beller, S. (2011). The cultural constitution of cognition: taking the anthropological perspective. Frontiers in Psychology, 2, 1-6. doi: 10.3389/ fpsyg.2011.00067.

Birch, S. A. J. y Bloom, P. (2003). Children are cursed: An asymmetric bias in mental-state attribution. Psychological Science, 14(3), 283-286. doi: 10.1111/14679280.03436 
Birch, S. A. J. y Bloom, P. (2007). The curse of knowledge in reasoning about false beliefs. Psychological Science, 18(5), 382-386. doi: 10.1111/j.1467-9280.2007.01909.x.

Brown, A. L., Kane, M. J. y Echols, C. H. (1986). Young children's mental models determine analogical transfer across problems with a common goal structure. Cognitive Development, 1(2), 103-121. doi: 10.1016/ S0885-2014(86)80014-4.

Burgoon, E. M., Henderson, M. D. y Markman, A. B. (2013). There are many ways to see the forest for the trees: A tour guide for abstraction. Perspectives on Psychological Science, 8(5), 501-520. doi: $10.1177 / 1745691613497964$.

Camarata, S. y Woodcock, R. (2006). Sex differences in processing speed: developmental effects in males and females. Intelligence, 34, 231-252. doi: 10.1016/j.inte11.2005.12.001.

Clements, A. M., Rimrodt, S. L., Abel, J. R., Blankner, J. G., Mostofsky, S. H. y Pekar, J. J. (2006). Sex differences in cerebral laterality of language and visuospatial processing. Brain Lang, 98, 150-158. doi: 10.1016/j. bandl.2006.04.007.

Colom, R., Espinosa, M. J., Abad, F. y García, L. F. (2000). Negligible sex differences in general intelligence. Intelligence, 28(1), 57-68. doi: 10.1016/S01602896(99)00035-5.

Dekker, S., Krabbendam, L., Aukje, A., deGroot, R. y Jolles, J. (2013). Coding task performance in early adolescence: a large-scale controlled study into boy-girl differences. Frontiers in Psychology, 4, 1-6. doi: 10.3389/ fpsyg.2013.00550.

De Neys, W. (2006). Dual processing in reasoning: Two systems but one reasoner. Psychological Science, 17(5), 428-433. doi: 10.1111/j.1467-9280.2006.01723.x.

Evans, J. (2003). In two minds: Dual process accounts of reasoning. Trends in Cognitive Sciences, 7(10), 454-459. doi:10.1016/j.tics.2003.08.012.

Evans, J. (2008). Dual-processing accounts of reasoning, judgment, and social cognition. Annual Review of Psychology, 59, 255-278. doi: 10.1146/annurev. psych.59.103006.093629.

Evans, J. y Stanovich, K. (2013). Dual-process theories of higher cognition: advancing the debate. Perspectives on Psychological Science, 8(3), 223-241. doi: $10.1177 / 1745691612460685$.

Finkel, D., Reynolds, C. A., McArdle, J. J. y Pedersen, N. L. (2005). The longitudinal relationship between processing speed and cognitive ability: Genetic and environmental influences. Behavior Genetics, 35(5), 535-549. doi:10.1007/s10519-0053281-5.
Galián, M. D., Carranza, J. A., Escudero, A. J., Ato, M. y Ato, E. (2006). Diferencias individuales en la competencia lingüística de los sujetos diferenciales y expresivos. Psicothema, 18(1), 37-42. Recuperado de http://www. psicothema.com/psicothema.asp?id=3173

Gardner, H. (2001). Estructura de la mente. La teoría de las inteligencias múltiples. México: Fondo de Cultura Económica.

Gilovich, T., Griffin, D. y Kahneman, D. (Eds.) (2002). Heuristics and biases: The psychology of intuitive judgment. Cambridge: Cambridge University Press.

Grissom, R. J. y Kim, J. J. (2005). Effect sizes for research: A Broad practical approach. Mahwah, NJ: Lawrence Erlbaum Associates.

Grissom, R. J. y Kim, J. J. (2012). Effect sizes for research: Univariate and Multivariate Applications. Londres: Routledge.

Halpern, D. F. (2004). A cognitive taxonomy for sex differences in cognitive abilities. Current Directions in Psychological Science, 13, 135-139. doi: 10.1111/j.09637214.2004.00292.x.

Halpern, D. F., Benbow, C. P., Geary, D. C., Gur, R. C., Shibley Hyde, J. y Gernsbacher, M. A. (2007). The science of sex differences in Science and Mathematics. Psychological science in the public interest, 8(1), 1-51. doi: 10.1111/j.1529-1006.2007.00032.x.

Herbert, J. y Hayne, H. (2000). Memory retrieval by 18-30-month-olds: age-related changes in representational flexibility. Developmental Psychology, 36(4), 473-484. doi:10.1037//0012-1649 36.4.473.

Hunt, E. y Carlson, J. (2007). Considerations relating to the study of Group Differences in Intelligence. Perspectives on Psychological Science, 2(2), 194-213. doi: 10.1111/j.1745-6916.2007.00037.x.

Johnson, W., Carothers, A. y Deary, I. A. (2008). Sex differences in variability in general intelligence: A new look at the old question. Perspectives on Psychological Science, 3(6), 518-531. doi: 10.1111/j.1745-6924.2008.00096.x.

Johnson-Laird, P. (1983). Mental models: Towards a cognitive Science of language, inference, and consciousness. Cambridge, Massachusetts: Harvard University Press.

Johnson-Laird, P. (1995). Mental models, deductive reasoning and the brain. En M. S. Gazzaniga (ed.), The cognitive neurosciences (pp. 999-1008). Cambridge, MA: MIT Press.

Johnson-Laird, P. (2006). How we reason. New York, NY: Oxford University Press. 
Johnson-Laird, P. (2010). Mental models and human reasoning. Pnas, 107(43), 18243-18250. doi: 10.1073/ pnas.1012933107.

Johnson-Laird, P. y Byrne, R. (1991). Deduction. Essays in cognitive psychology. Hillsdale, NJ: Lawrence Erlbaum.

King, J. W. y Suzman, R. (2008). Prospects for improving cognition throughout the life course. Psychological science in the public interest, 9(1), 1-3. doi: 10.1111/j.15396053.2009.01033.x.

Kirkham, N. Z., Cruess, L. y Diamond, A. (2003). Helping children apply their knowledge to their behavior on a dimension-switching task. Developmental Science, 6(5), 449-467. doi: 10.1111/1467-7687.00300.

Knight, N. y Nisbett, R. E. (2007). Culture, class and cognition: Evidence from Italy. Journal of Cognition and Culture, 7, 283-291. doi: 10.1163/156853707X208512.

Macbeth, G., Razumiejczyk, E. y Crivello, M. D. C. (2014). Efecto de la distorsión introspectiva sobre los errores de superficie en el razonamiento lógico. Boletín de Psicología, 11(2), 25-43. Recuperado de http://www.uv.es/ seoane/boletin/previos/N111-2.pdf.

Macbeth, G., Razumiejczyk, E., Crivello, M. D. C., Bolzán, C., Pereyra Girardi, C. I. y Campitelli, G. (2014). Mental models for the negation of conjunctions and disjunctions. Europe's Journal of Psychology, 10(1), 135-149. doi: 10.5964/ejop.v10i1.696.

Maccoby, E. E. y Jacklin, C. N. (1974). The psychology of sex differences. London: Oxford University Press.

Macpherson, R. y Stanovich, K. (2007). Cognitive ability, thinking dispositions, and instructional set as predictors of critical thinking. Learning and individual differences, 17, 115-127. doi:10.1016/j.lindif.2007.05.003.

Matsuyoshi, D., Osaka, M. y Osaka, N. (2014). Age and individual differences in visual working memory deficit induced by overload. Frontiers in Psychology, 5, 1-7. doi: 10.3389/fpsyg.2014.00384.

Medin, D. L., Bennis, W. M. y Chandler, M. (2010). Culture and the home-field disadvantage. Perspectives on Psychological Science, 5(6), 708-713. doi: $10.1177 / 1745691610388772$.

Mills, C. J., Ablard, K. E. y Stumpf, H. (1993). Gender differences in academically talented young students' mathematical reasoning: Patterns across age and subskills. Journal of Educational Psychology, 85, 340-346. doi: 10.1037/0022-0663.85.2.340.

Monserrat, M. y Valiña, M. D. (2002). Razonamiento deductivo: una aproximación al estudio de la disyunción. Revista de Psicología General y Aplicada, 55(2), 225 248. Recuperado de https://dialnet.unirioja.es/servlet/ articulo? codigo $=274687$
Newstead, S. E. (2000). Are there two different types of thinking? Behavioral and Brain Sciences, 3(5), 690-691. doi: 10.1017/S0140525X0049343X.

Newstead, S. E., Handley, S. J., Harley, C., Wright, H. y Farrelly, D. (2004). Individual differences in deductive reasoning. The quarterly journal of experimental psycho$\log y$, 57(1), 33-60. doi: 10.1080/02724980343000116.

Nyberg, L., Maitland, S. B., Rönnlund, M., Bäckman, L., Dixon, R. A., Wahlin, Å. y Nilsson, L. G. (2003). Selective adult age differences in an age-invariant multifactor model of declarative memory. Psychology and aging, 18(1), 149-160. doi: 10.1037/0882-7974.18.1.149.

Nyberg, L., Lövde'n, M., Riklund, K., Lindenberger, U. y Bäckman, L. (2012). Feature Review Memory aging and brain maintenance. Trends in Cognitive Sciences, 16(5), 292-305. doi:10.1016/j.tics.2012.04.005.

Peters, E., Hess, T. M., Västfjäll, D. y Auman, C. (2007). Adult age differences in dual information processes: implications for the role of affective and deliberative processes in older adults' decision making. Perspectives on Psychological Science, 2(1), 1-23. doi: 10.1111/j.17456916.2007.00025.x.

Rattermann, M. J., Gentner, D. y DeLoache, J. (1990). The effects of familiar labels on young children's performance in an analogical mapping task. En Proceedings of the Twelfth Annual Conference of the Cognitive Science Society (pp. 22-29). Hillsdale, NJ: Lawrence Erlbaum Associates.

Restrepo, J. E. (2015). Correlatos cognitivos y neuropsicológicos de los cinco grandes: una revisión en el área de la neurociencia de la personalidad. Pensando Psicología, 11(18), 107-127. doi: 10.16925/pe.v11i18.1004.

Rips, L. (1989). The psychology of Knights and Knaves. Cognition, 31, 85-116. doi: 10.1016/0010-0277(89)90019-X.

Rips, L. (1994). The psychology of proof. Cambridge, MA: MIT Press.

Robinson, N. M., Abbott, R. D., Berninger, V. W. y Busse, J. (1996). The structure of abilities in mathematically precocious young children: Gender similarities and differences. Journal of Educational Psychology, 88, 341-352. doi: 10.1037/0022-0663.88.2.341.

Rushton, J. P. y Jensen, A. R. (2005). Thirty years of research on race differences in cognitive ability. Psychology, Public Policy, and Law, 11(2), 235-294. doi: 10.1037/1076-8971.11.2.235.

Schank, R. C. y Abelson, R. P. (1995). Knowledge and memory: the real story. En R. S. Wyer Jr. (Ed.), Knowledge and memory: the real story, Hillsdale: Lawrence Erlbaum Associates. 
Schmidt, N. (1996). Statistical significance testing and cumulative knowledge in psychology: Implications for training of researchers. Psychological methods, 1(2), 115-129. doi: 10.1037/1082-989X.1.2.115.

Schroyens, W., Schaeken, W., Fias, W. y d'Ydewalle, G. (2000). Heuristic and analytic processes in propositional reasoning with negatives. Journal of Experimental Psychology: Learning, Memory, and Cognition, 26(6), 1713-1734. doi: 10.1037/0278-7393.26.6.1713

Seoane, G., Valiña, M. D., Rodríguez, M. S., Montserrat, M. y Ferraces, M. J. (2007). Diferencias individuales en razonamiento hipotético-deductivo: importancia de la flexibilidad y de las habilidades cognitivas. Psicothema, 19(2), 206-211. Recuperado de http://www.psicothema.com/psicothema. asp?id=3349.

Son, J., Smith, L B., Goldstone, R. L. y Leslie, M. (2012). The importance of being interpreted: grounded words and children's relational reasoning. Frontiers in Psychology, 3, 1-12. doi: 10.3389/fpsyg.2012.00045.

Sowell, T. (2005). Black rednecks and White liberals. San Francisco: Encounter Books.

Spelke, E. S. (2005). Sex differences in intrinsic aptitude for mathematics and science: A critical review. American Psychologist, 60, 950-958. doi: 10.1037/0003066X.60.9.950.

Stanovich, K. E. (2012). On the distinction between rationality and intelligence: Implications for understanding individual differences in reasoning. En K. J. Holyoak y R. G. Morrison (Eds.), The Oxford handbook of thinking and reasoning (pp. 343-365). New York: Oxford University Press.

Stanovich, K. E. y West, R. F. (1997). Reasoning independently of prior belief and individual differences in actively open-minded thinking. Journal of Educational Psychology, 89(2), 342-357. doi: 10.1037/00220663.89.2.342
Stanovich, K. E. y West, R. F. (1998). Individual differences in rational thought. Journal of experimental psychology: general, 127(2), 161-188. doi: 10.1037/ 00963445.127.2.161

Stanovich, K. y West, R. (2000). Individual differences in reasoning: Implications for the rationality debate? $\mathrm{Be}$ havioral and brain sciences, 23, 645-726. doi: 10.1017/ S0140525X00003435

Stanovich, K. E., West, R. y Toplak, M. E. (2013). Myside bias, rational thinking, and intelligence. Current $\mathrm{Di}$ rections in Psychological Science, 22(4), 259-264. doi: 10.1177/0963721413480174

Sternberg, R. J., Grigorenko, E. L. y Kidd, K. K. (2005). Intelligence, race, and genetics. American Psychologist, 60(1), 46-59. doi: 10.1037/0003-066X.60.1.46.

Sternberg, R. J., Grigorenko, E. L. y Zhang, L. (2008). Styles of learning and thinking matter in instruction and assessment. Perspectives on Psychological Science, 3(6), 486-506. doi: 10.1111/j.1745-6924.2008. 00095.x.

Weber, E. U. y Morris, M. W. (2010). Culture and judgment and decision making: the constructivist turn. Perspectives on Psychological Science, 5(4), 410-419. doi: 10.1177/17456916103755.

Wild-Wall, N., Falkenstein, M. y Gajewski, P. D. (2011). Age-related differences in working memory performance in a 2-back task. Frontiers in Psychology, 2, 1-12. doi: 10.3389/fpsyg.2011.00186.

William L. J., Dunlop J. P. y Abdi, H. (2012). Effect of age on variability in the production of text-based global inferences. Plos One, 7(5), 1-9. doi: 10.1371/journal. pone.0036161. 